\title{
Caracterización experimental y modelación de una bomba de calor reversible para un vehículo eléctrico
}

\author{
Experimental characterization and modelling of a reversible \\ heat pump for electric automotive application
}

\author{
Cristian Cuevas $^{1 *} \quad$ Vincent Lemort ${ }^{2}$
}

Recibido 11 de enero de 2016, aceptado 14 de abril de 2016

Received: January 11, 2016 Accepted: April 16, 2016

\begin{abstract}
RESUMEN
El sistema de aire acondicionado y de calefacción en los automóviles se utiliza principalmente por motivos de seguridad para garantizar que el conductor esté concentrado y con buena visibilidad durante la conducción. Para la calefacción, en los vehículos convencionales, se cuenta con una energía térmica considerable y a una excelente temperatura, esto debido al calor disipado por el motor a combustión. Los vehículos eléctricos no cuentan con esta fuente de calor, por lo que se debe suplir por algún sistema auxiliar como resistencias eléctricas o una bomba de calor. En el estudio que se desarrolla en esta investigación se propone utilizar una bomba de calor para la calefacción del automóvil. El objetivo que se plantea en este estudio es validar experimentalmente un principio de calefacción para automóviles eléctricos basado en una de bomba de calor reversible y desarrollar un modelo de simulación para explorar su desempeño a diferentes condiciones de operación. La bomba de calor se logró ensayar a temperaturas de hasta $-10^{\circ} \mathrm{C}$, obteniéndose COP superiores a 2,0 en todo el rango experimental. La modelación de la bomba de calor permite establecer que esta es capaz de funcionar en condiciones aceptables de hasta $-10{ }^{\circ} \mathrm{C}$, con un COP del orden de 1,48 y un calor transferido por el radiador de $4,1 \mathrm{~kW}$, con un flujo de aire impulsado a la cabina a $45^{\circ} \mathrm{C}$, lo que permite mantenerla a temperaturas por sobre los $20^{\circ} \mathrm{C}$.
\end{abstract}

Palabras clave: Bomba de calor, reversible, vehículo eléctrico, caracterización experimental, modelado.

\section{ABSTRACT}

Automotive air conditioning and heating are mainly used for security, to facilitate driving and to guarantee a good visibility. In conventional vehicles, heating is provided by the cooling system of the internal combustion engine. Electric vehicles do not have this kind of heating source, thus heating must be provided by electric resistances or heat pumps. In this article this last technology is considered in this article as a heating system for vehicles. The aim of this study is to validate experimentally a proposed heating principle for vehicles based on a reversible heat pump and to develop and validate a semiempirical model used to explore the heat pump performance under different working conditions. The heat pump is characterized at temperatures as low as $-10^{\circ} \mathrm{C}$, by obtaining COPs higher than 2,0. The heat pump simulation shows that the heat pump gives a COP of 1,48 at an ambient temperature of $-10^{\circ} \mathrm{C}$, by supplying $4,1 \mathrm{~kW}$ to the vehicle cabin at $45^{\circ} \mathrm{C}$ and keeping a temperature over $20^{\circ} \mathrm{C}$ at the vehicle cabin.

Keywords: Heat pump, reversible, electric vehicle, experimental characterization, modelling.

1 Departamento de Ingeniería Mecánica. Universidad de Concepción. Edmundo Larenas 219. Casilla 160-C. Concepción, Chile E-mail: crcuevas@udec.cl

2 Thermodynamics Laboratory. University of Liège. Campus du Sart Tilman. Bâtiment B49. B-4000 Liège. Belgium.

E-mail: vincent.lemort@ulg.ac.be

* Autor de correspondencia 


\section{INTRODUCCIÓN}

Los vehículos eléctricos no gozan de una fuente de calor como los vehículos convencionales para utilizarla como fuente de calefacción de la cabina. Una alternativa es utilizar resistencias eléctricas, lo que es menos eficiente que otro tipo de tecnología como, por ejemplo, las bombas de calor. En la industria automóvil existen numerosas investigaciones relacionadas con el uso de bombas de calor para calefacción. Las referencias [1-3] analizan el uso de bombas de calor que utilizan una fuente de calor dual: aire ambiente y calor residual del sistema. Suzuki e Ishii [4] realizan un estudio similar al desarrollado en este estudio, obteniendo un $\mathrm{COP}$ de 2,3 y un flujo de calor de $2,3 \mathrm{~kW}$ para una bomba de calor operando a $-10{ }^{\circ} \mathrm{C}$.

En el estudio que se presenta en este trabajo se analiza una bomba de calor reversible, la que solo se analiza en modo calefacción. Esta utiliza como fuente de calor el aire ambiente. El sistema propuesto está constituido por componentes convencionales utilizados en un sistema de aire acondicionado de automóvil, al que se le ha agregado un circuito de agua con etileno glicol con un intercambiador de calor de placas y una válvula cuatro vías. En modo aire acondicionado el sistema trabaja como un sistema convencional y en modo calefacción se utiliza el intercambiador de calor a placas como condensador. El calor que disipa el condensador es enviado por medio del radiador del módulo de aire acondicionado a la cabina del automóvil.

\section{DESCRIPCIÓN DEL SISTEMA}

El principio de funcionamiento de la bomba de calor se ilustra en la Figura 1.

Este sistema está pensado para que pueda funcionar cuando el vehículo opera con el motor a combustión, con el motor eléctrico y en modo aire acondicionado y en modo calefacción.

Para implementar este sistema se utilizan equipos convencionales utilizados en el sistema de aire acondicionado de vehículos, al cual se le agrega una válvula cuatro vías para invertir el ciclo, un condensador de placas y una válvula de expansión para el intercambiador exterior. La Figura 2 muestra los componentes utilizados en ambos modos.

El fluido refrigerante utilizado es R134a y en el circuito del condensador, usado en modo calefacción, se usa una mezcla de agua con etileno glicol en una proporción de 50/50 en masa.

\section{Compresor}

El compresor es una máquina de plato oscilante de 7 pistones con un volumen barrido de $161,3 \mathrm{~cm}^{3}$. Este compresor fue instalado es un sistema basculante para medir su torque y además se mide su velocidad

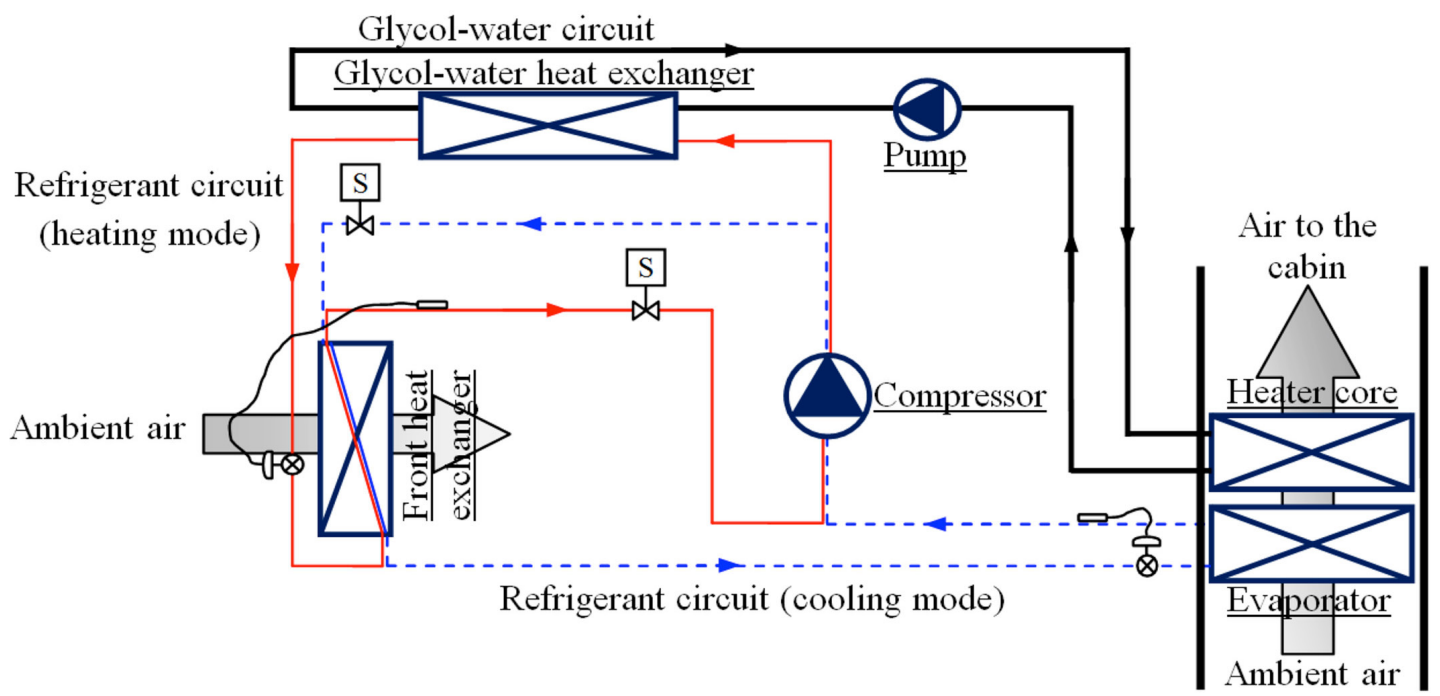

Figura 1. Principio de funcionamiento de la bomba de calor. 


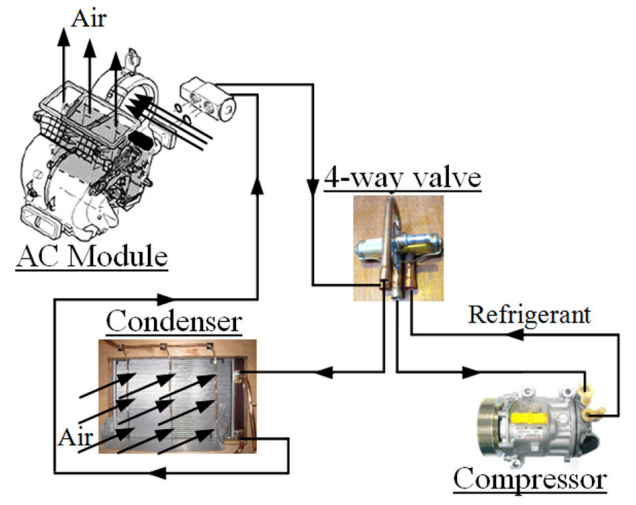

(a) Cooling mode

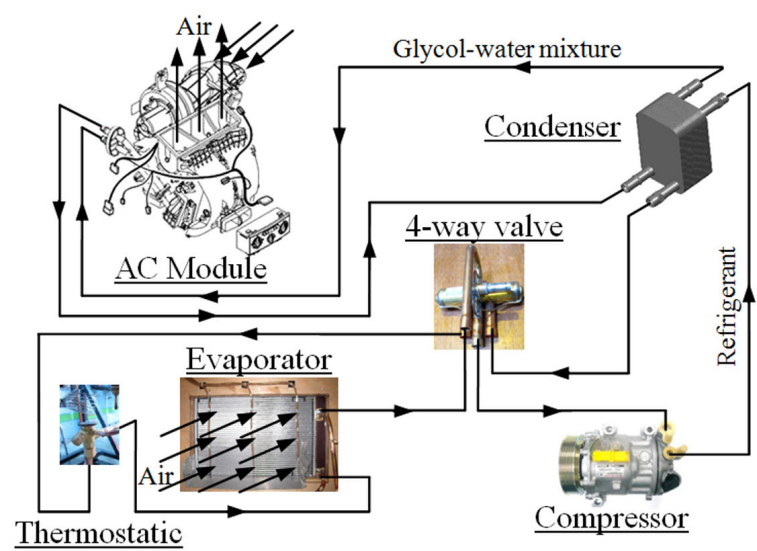

(b) Heating mode

expansion

valve

Figura 2. Componentes utilizados para caracterizar la bomba de calor.

de giro para determinar su potencia al eje, tal como se indica en Cuevas et al. [5] (ver Figura 3). El compresor es instalado dentro de un calorímetro para determinar sus pérdidas al ambiente.

\section{Intercambiador de calor exterior}

El intercambiador de calor exterior es un dispositivo de tubos planos y aletas con persianas (ver Figura 4). Este trabaja como evaporador en modo calefacción y como condensador en modo aire acondicionado.

Del lado aire se instalan nueve termocuplas a la entrada y a la salida para determinar su calor transferido.

\section{Intercambiador de calor agua/glicol}

El intercambiador de calor usado como condensador en modo calefacción es un dispositivo de placas de aluminio, que se muestra en la Figura 5, y cuyas dimensiones son $100 \mathrm{~mm}$ de ancho, $175 \mathrm{~mm}$ de alto y $75 \mathrm{~mm}$ de fondo. En los vehículos, el peso de los componentes es importante ya que esto incide

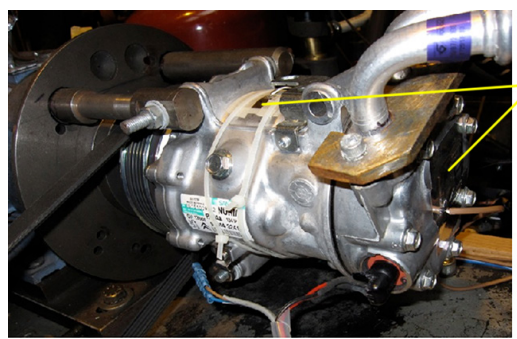

Termocuplas de superficie

Figura 3. Compresor de plato oscilante.

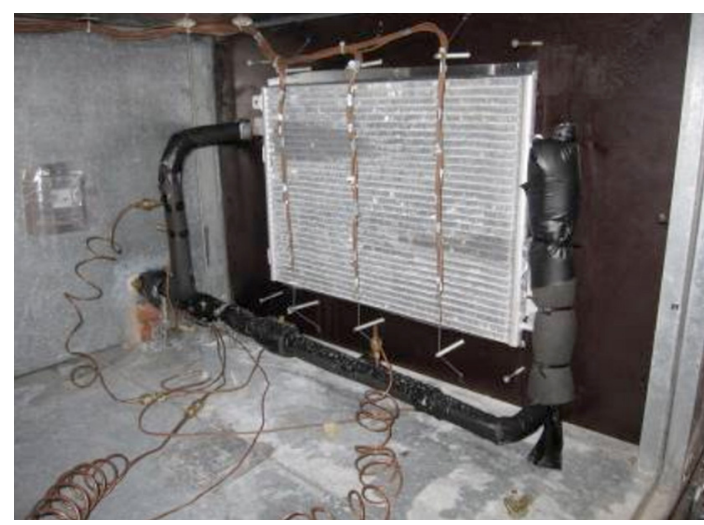

Figura 4. Intercambiador de calor exterior.

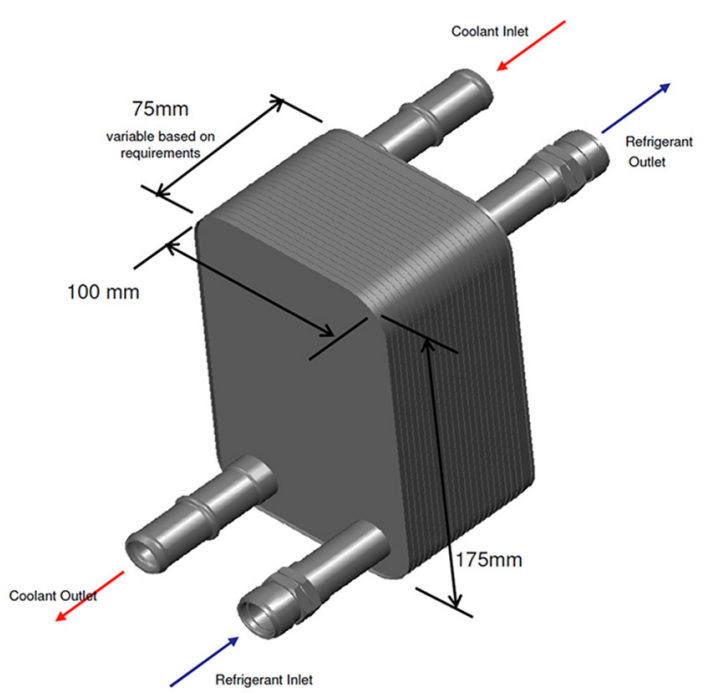

Figura 5. Intercambiador de calor de placas. 
directamente sobre el consumo de combustible o eléctrico, es por esto que se usa un condensador de aluminio.

\section{Módulo de aire acondicionado}

El módulo de aire acondicionado es un aparato convencional compuesto por un ventilador, un evaporador con su válvula de expansión y un radiador (ver Figura 6), además del sistema de regulación manual de los flujos de aire y de temperatura.

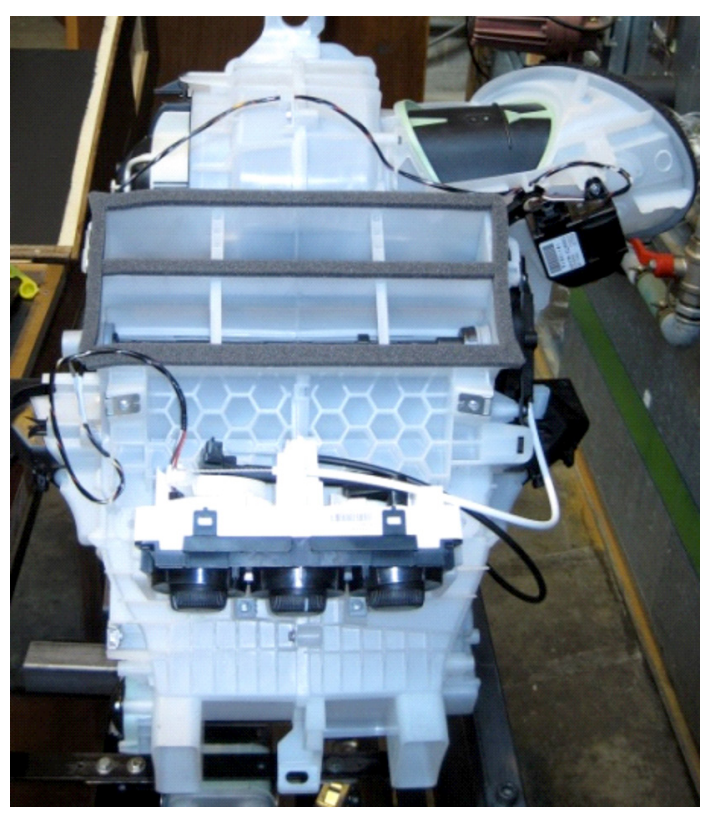

Figura 6. Módulo de aire acondicionado.

\section{RESULTADOS EXPERIMENTALES}

El sistema es caracterizado con 61 ensayos en modo bomba de calor y con 19 ensayos en modo aire acondicionado. En este artículo se desarrollan solamente los resultados relativos al modo calefacción.

Los ensayos se estabilizan durante un período suficientemente largo para alcanzar un régimen estacionario. Una vez alcanzado este régimen se toman promedios durante un período estable de al menos unos 30 minutos. Las mediciones permiten determinar los flujos de energía por más de un balance de energía. Por ejemplo, en el caso del condensador, el calor transferido se puede determinar del lado refrigerante y del lado de la mezcla de agua con etileno glicol, por lo que la comparación de estos dos valores permiten validar la metodología de medición y chequear la precisión de los balances de energía. La Figura 7 muestra esta comparación obteniéndose una diferencia considerada en este estudio como aceptable.

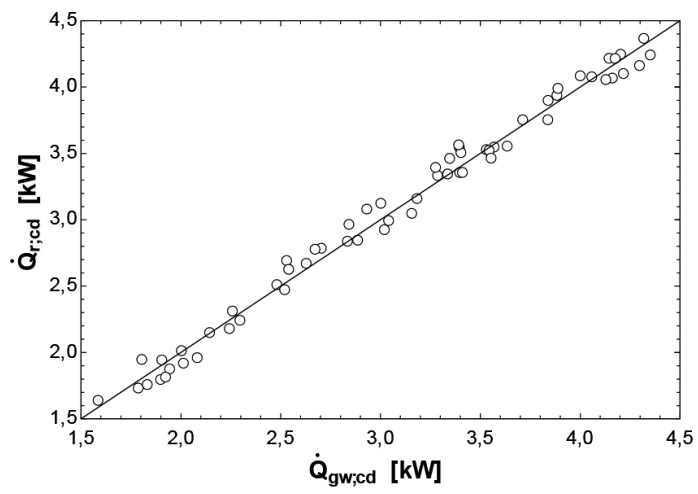

Figura 7. Verificación de los balances de energía en el condensador.

La Figura 8 muestra el COP del sistema para todos los ensayos. En este caso, y a modo de ejemplo, se analiza el punto marcado en la figura para una temperatura exterior del orden de $-9,8^{\circ} \mathrm{C}$, para el que se obtiene un COP del orden de 2,8 .

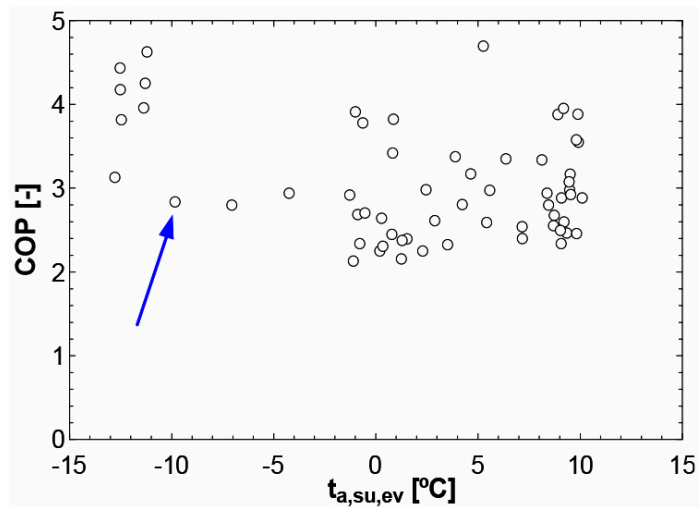

Figura 8. COP del sistema.

Para este punto el radiador de la unidad de aire acondicionado es capaz de entregar un flujo de calor del orden de $1,9 \mathrm{~kW}$, tal como se ilustra en la Figura 9, a una temperatura de $33,5^{\circ} \mathrm{C}$, como se ilustra en la Figura 10. 


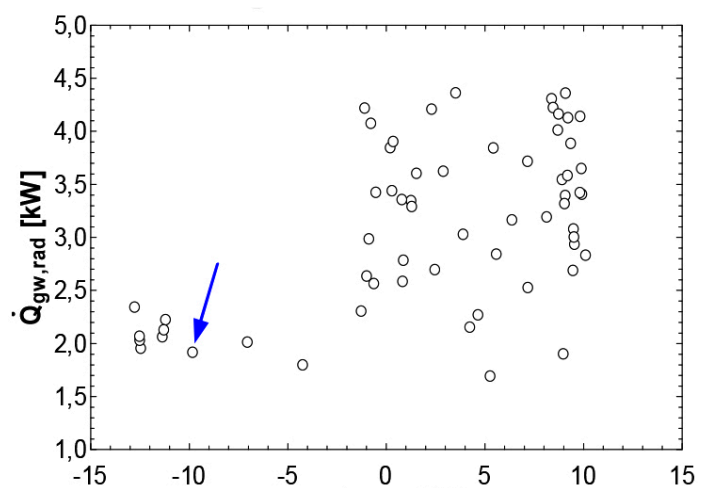

Figura 9. Flujo de calor entregado a la cabina.

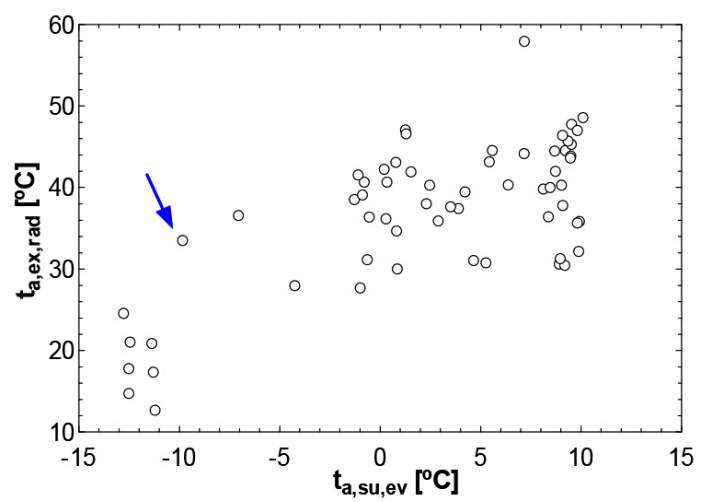

Figura 10. Temperatura del aire impulsado a la cabina.

Durante la campaña de mediciones se realizaron numerosos ensayos que permitieron caracterizar los componentes del sistema para poder posteriormente realizar los modelos de simulación.

\section{MODELACIÓN}

Los resultados obtenidos de la campaña de mediciones se utilizan para desarrollar un modelo de simulación semiempírico de la bomba de calor y realizar un análisis paramétrico que permita analizar su desempeño bajo diferentes condiciones de operación. El modelo de la bomba de calor consta de cuatro submodelos de los siguientes equipos: compresor, condensador, evaporador y radiador, los que son desarrollados en el software EES [6]. Los modelos de estos equipos se describen a continuación.

\section{Modelo del compresor}

El compresor es modelado como se indica en [5]. El modelo considera las pérdidas de carga en las válvulas de admisión y descarga, los intercambios de calor del refrigerante a la entrada y salida, el intercambio de calor con el medio ambiente y el proceso de compresión. Las principales salidas del modelo del compresor son el flujo de refrigerante, la potencia al eje y la temperatura del refrigerante a la salida.

El flujo de refrigerante se calcula a partir de la ecuación (1):

$$
\dot{M}_{r, c p}=\frac{\varepsilon_{v} \cdot \dot{V}_{s}}{v_{r, s u 1, c p}}
$$

Donde el rendimiento volumétrico se determina como:

$$
\varepsilon_{v}=1-C_{f} \cdot\left(r_{v}-1\right)=1-C_{f} \cdot\left(\frac{v_{r, s u 1, c p}}{v_{r, e x 1, c p}}-1\right)
$$

La potencia al eje del compresor se determina como:

$$
\dot{W}_{c p}=\dot{W}_{i n, c p}+\dot{W}_{l o s s, c p}
$$

Donde las pérdidas mecánicas del compresor se modelan como:

$$
\begin{gathered}
\dot{W}_{\text {loss }, c p}=\alpha \cdot \dot{W}_{\text {in }, c p}+\dot{W}_{\text {loss } 0, c p} \cdot\left(\frac{N}{N_{\text {ref }}}\right)+ \\
\dot{W}_{\text {loss } 1, c p} \cdot\left(\frac{N}{N_{\text {ref }}}\right)^{2}
\end{gathered}
$$

Los parámetros del modelo se identifican comparando las salidas del modelo con los valores obtenidos de la caracterización experimental. La Figura 11 muestra un ejemplo de esta comparación para la potencia al eje del compresor simulada y medida para todos los puntos de los ensayos realizados en modo calefacción y enfriamiento, es decir, para un total de 80 ensayos. Para tener una idea del error se determina la raíz del error cuadrático medio dando un valor de $3,0 \%$.

\section{Modelo del condensador}

El condensador es modelado utilizando una aproximación de tres zonas [7]. Las ecuaciones principales para determinar el calor transferido en cada zona son las siguientes: 


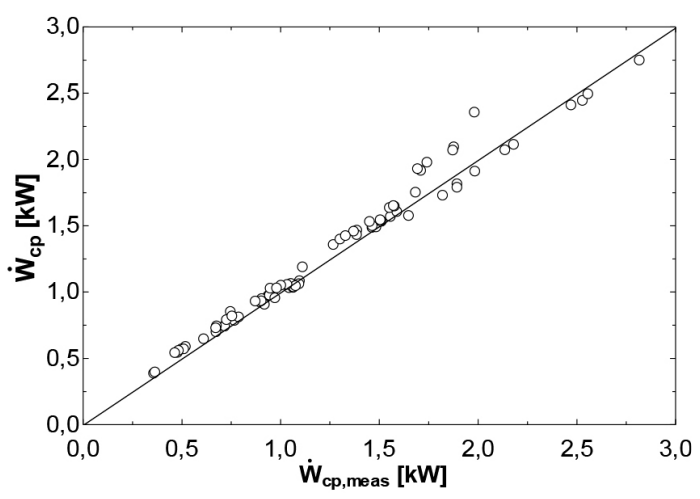

Figura 11. Comparación potencia del compresor.

$$
\begin{aligned}
& \dot{Q}_{r, z i, c d}=\dot{M}_{r, z i, c d} \cdot\left(h_{r, s u, z i, c d}-h_{r, e x, z i, c d}\right) \\
& \dot{Q}_{w, z i, c d}=\dot{M}_{w, z i, c d} \cdot\left(h_{w, e x, z i, c d}-h_{w, s u, z i, c d}\right) \\
& Q_{z i, c d}=\varepsilon_{z i, c d} \cdot \dot{C}_{\min , z i, c d} \cdot\left(t_{r, s u, z i, c d}-t_{w, s u, z i, c d}\right)
\end{aligned}
$$

La transferencia de calor es determinada con el método $\varepsilon$-NTU:

$$
\begin{gathered}
N T U_{z i, c d}=\frac{U \cdot A_{z i, c d}}{\dot{C}_{\min , z i, c d}} \\
\frac{1}{(A \cdot U)_{z i, c d}}=\frac{1}{h_{i, z i, c d} \cdot A_{i, z i, c d}}+ \\
R_{w}+\frac{1}{h_{o, z i, c d} \cdot A_{o, z i, c d}}
\end{gathered}
$$

Donde los coeficientes convectivos se determinan como:

$$
h=h_{n o m} \cdot\left(\frac{\dot{M}}{\dot{M}_{\text {nom }}}\right)^{n}
$$

Globalmente, considerando las tres zonas, para los flujos de calor se tendrá que:

$$
\begin{gathered}
\dot{Q}_{w, c d}=\dot{Q}_{w, s h, c d}+\dot{Q}_{w, t p, c d}+\dot{Q}_{w, s c, c d} \\
\dot{Q}_{r, c d}=\dot{Q}_{r, s h, c d}+\dot{Q}_{r, t p, c d}+\dot{Q}_{r, s c, c d}
\end{gathered}
$$

El área de transferencia de calor del condensador es igual a la suma de las áreas ocupadas por cada una de las zonas:

$$
A_{c d}=A_{s h, c d}+A_{t p, c d}+A_{s c, c d}
$$

Este modelo permite cuantificar las áreas ocupadas

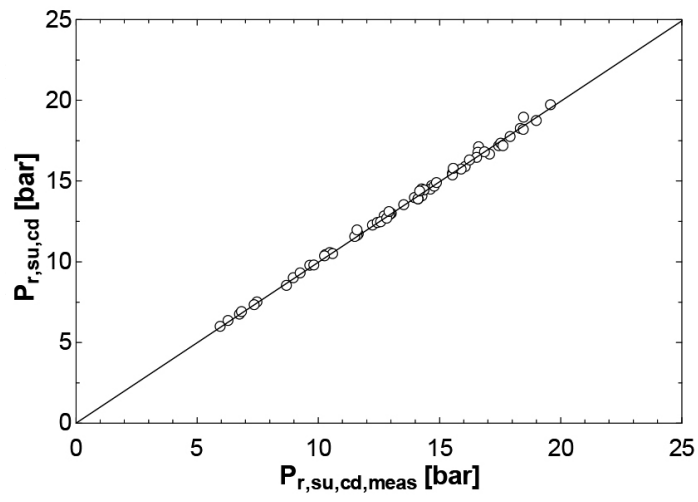

Figura 12. Comparación entre la presión de entrada del condensador simulada y medida.

\section{Modelo del evaporador}

El evaporador es modelado en forma similar al condensador, con la única diferencia que en este caso se modela considerando una sola zona. Además se agrega un modelo de pérdida de carga del lado refrigerante, debido a que este equipo presenta una pérdida de carga considerable, que puede llegar hasta 1,5 bar. Esto se debe a que este equipo está optimizado para funcionar como condensador, y al momento de invertir el ciclo y hacerlo trabajar como evaporador, se producen pérdidas de carga excesivas a la entrada de este. La pérdida de carga es modelada con la siguiente expresión:

$$
\Delta P_{r}=\Delta P_{r, n} \cdot\left(\frac{\dot{M}_{r}}{\dot{M}_{r, n}}\right)^{2-p}
$$

Este modelo no considera ni la condensación ni el congelamiento de la humedad del aire sobre el evaporador, ya que este se ensayó con aire seco.

La Figura 13 muestra la comparación de la potencia que predice el modelo y la medida, se observa una buena correlación entre ambas. La raíz del error cuadrático medio es de un 1,6\%.

\section{Modelo del radiador}

El radiador es modelado en forma similar al modelo del condensador, con la diferencia que en este caso no hay cambio de fase. 


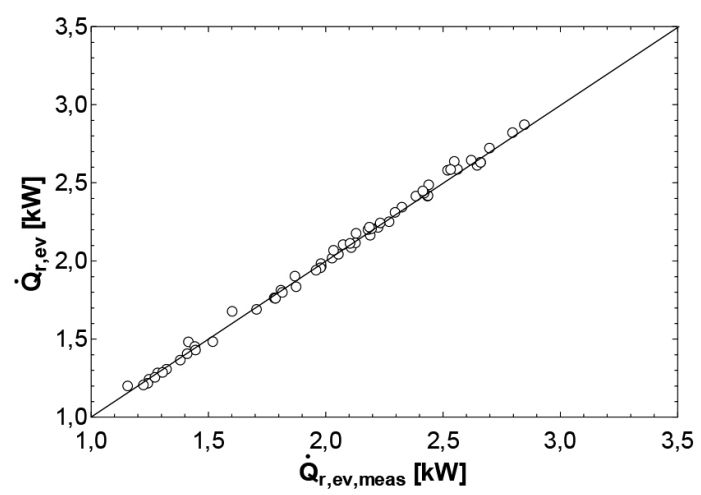

Figura 13. Comparación entre la potencia del evaporador simulada y medida.

La principal salida del modelo del radiador es el flujo de calor transferido, el que se compara con el flujo medido en la Figura 14. La raíz del error cuadrático medio es de un $4,7 \%$.

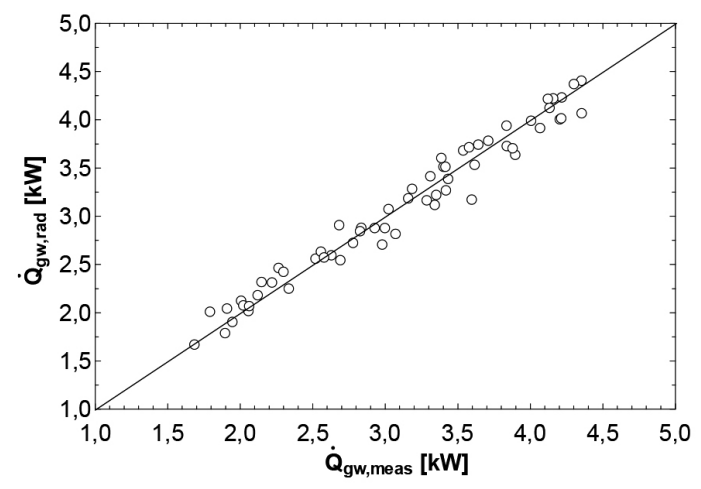

Figura 14. Comparación entre la potencia del radiador simulada y medida.

\section{Modelo de la bomba de calor}

El modelo de la bomba de calor se obtiene conectando los modelos de los diferentes componentes, lo que da origen al modelo que se muestra en la Figura 15.

Este modelo asume que el compresor impone el flujo de refrigerante que circula en la instalación y la temperatura del refrigerante en su descarga, que el conjunto radiador-condensador imponen la presión de condensación y que el evaporador impone la presión de evaporación.

Considerando las interconexiones presentadas previamente y los modelos individuales, las entradas del modelo global se reducen a las siguientes:

Compresor: temperatura ambiente, velocidad de giro.

Condensador: flujo másico de la mezcla agua-etileno glicol, subenfriamiento del refrigerante (se supone que este está impuesto por la carga de refrigerante).

Evaporador: flujo másico del aire, temperatura de entrada del aire, sobrecalentamiento del refrigerante (se supone que este está impuesto por el modelo de la válvula de expansión).

Radiador: temperatura de entrada del aire.

Las principales salidas del modelo son el calor transferido en el radiador, la temperatura del aire impulsado por el módulo de aire acondicionado y la potencia del compresor. Estas permiten determinar el COP del sistema.

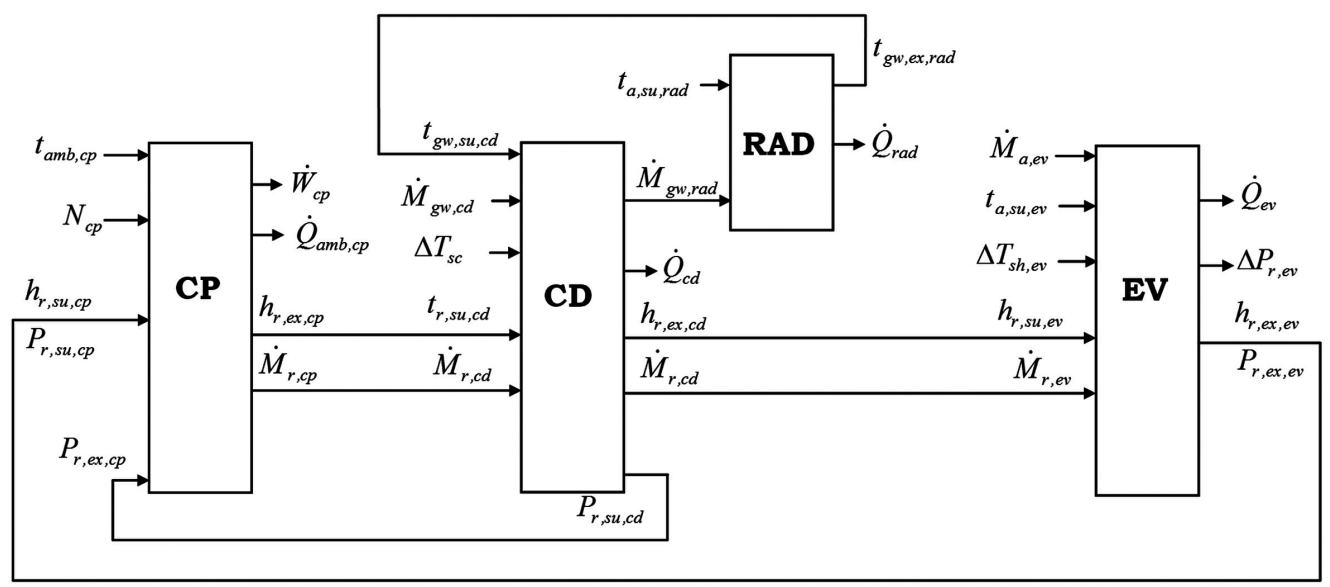

Figura 15. Modelo de la bomba de calor. 
La Figura 16 muestra la comparación de los resultados del COP del modelo y el COP medido de la bomba de calor. Se hace una diferencia entre una primera y una segunda campaña de mediciones. Los puntos que muestran mayor diferencia con respecto al modelo son aquellos que presentan una baja potencia al eje del compresor.

Esto se atribuye a la incertidumbre asociada a la determinación de la potencia al eje del compresor, la que tiene un error asociado a la medición del torque y otro a la velocidad de rotación, siendo la determinación del torque la más crítica, debido al torque parásito que introducen las líneas de succión y descarga del compresor, tal como se menciona en [5]. Para minimizar el efecto de este error se realizan calibraciones al comienzo y al térmico de cada campaña de mediciones. Determinando la raíz del error cuadrático medio se obtiene que para todos los puntos el error es de 7,9\%, y al excluir los puntos de la primera campaña se obtiene un error de $2,9 \%$.

\section{SIMULACIÓN DE LA BOMBA DE CALOR}

Una vez desarrollado el modelo de la bomba de calor se realiza una simulación del sistema completo para la siguiente condición de operación del vehículo: velocidad de aire frontal de $3 \mathrm{~m} / \mathrm{s}$, recirculación de aire de la cabina hacia el radiador de un 50\%, temperatura de impulsión de $45^{\circ} \mathrm{C}$ y temperaturas de aire ambiente de $5{ }^{\circ} \mathrm{C}, 0{ }^{\circ} \mathrm{C},-5^{\circ} \mathrm{C},-7,5^{\circ} \mathrm{C}$ y $-10{ }^{\circ} \mathrm{C}$. El flujo de aire impulsado a la cabina se deja flotante con un máximo de $470 \mathrm{~kg} / \mathrm{h}$ y se asume una temperatura de refrigerante límite de descarga del compresor de $130{ }^{\circ} \mathrm{C}$, la cual es impuesta para

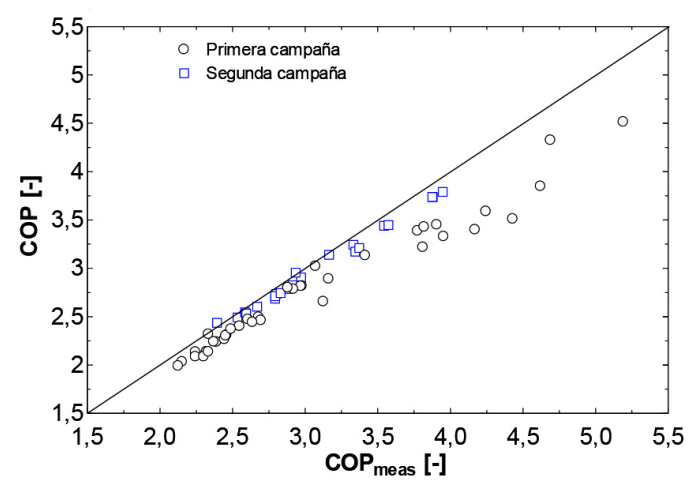

Figura 16. Comparación del COP de la bomba de calor. no tener problemas de carbonización del aceite. Se asume una temperatura de confort mínima en la cabina de $20^{\circ} \mathrm{C}$ y un coeficiente global de pérdidas de la cabina de $0,09 \mathrm{~kW} / \mathrm{K}$.

La Figura 17 muestra la temperatura de la cabina que se alcanza con este sistema y la velocidad que debería tener el compresor para impulsar un flujo de aire a $45^{\circ} \mathrm{C}$ a la cabina. En esta figura se observa que hasta $-10^{\circ} \mathrm{C}$ el sistema logra mantener prácticamente a $20^{\circ} \mathrm{C}$ la cabina, para lo cual el compresor debe girar a $4.100 \mathrm{rpm}$, la que es una velocidad que se puede alcanzar en este tipo de compresores.

Para estas condiciones se determina el COP del sistema, asumiendo un rendimiento del eventual inversor-motor eléctrico del compresor de un $90 \%$. En la Figura 18 se observa que el COP de la bomba de calor a $-10^{\circ} \mathrm{C}$, para una temperatura de la cabina de $20^{\circ} \mathrm{C}$, es del orden de 1,48 . En esta condición,

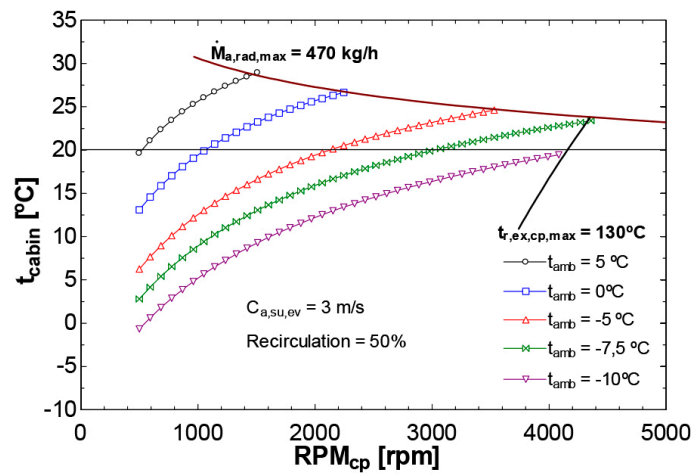

Figura 17. Temperatura de la cabina vs. velocidad compresor.

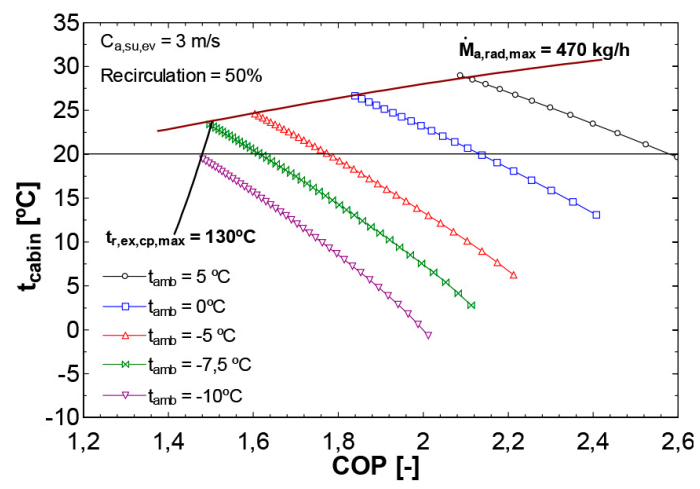

Figura 18. Temperatura de la cabina vs. COP del sistema. 
el radiador entrega un flujo de calor a la cabina del automóvil de 4,1 kW.

En relación a la potencia del motor eléctrico del compresor esta es del orden de $2,56 \mathrm{~kW}$ para la temperatura exterior de $-10{ }^{\circ} \mathrm{C}$, tal como se indica en la Figura 19.

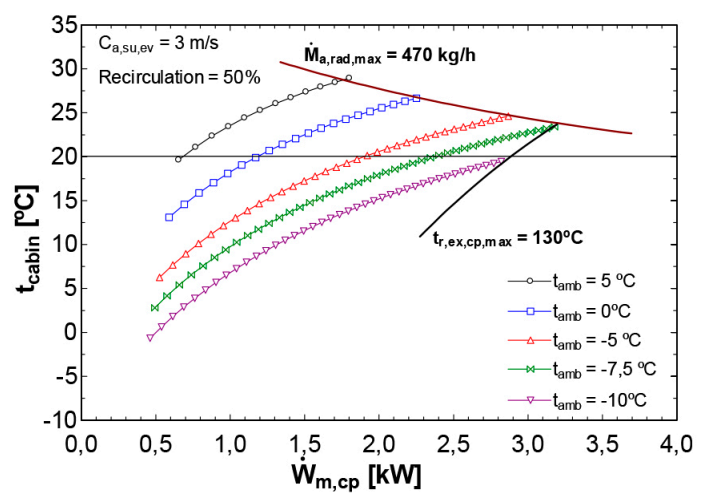

Figura 19. Potencia del motor eléctrico del compresor.

\section{CONCLUSIONES}

Se logró validar experimentalmente el sistema propuesto, sin evidenciarse inconvenientes técnicos. El sistema se logró caracterizar experimentalmente en modo calefacción y en modo aire acondicionado. Se ensayó en modo bomba de calor hasta temperaturas de $-10^{\circ} \mathrm{C}$ logrando COPs superiores a 2,0. Los resultados experimentales se utilizaron para generar y validar modelos de simulación semiempíricos que permitieron explorar el desempeño de la bomba de calor a diferentes condiciones de operación. En particular en la condición analizada se observa que con la bomba de calor se logran obtener temperaturas de la cabina iguales o superiores a $20^{\circ} \mathrm{C}$ hasta $-10^{\circ} \mathrm{C}$. Para ello se requiere que el compresor gire a $4.100 \mathrm{rpm}$, que el radiador entregue un flujo de calor de $4,1 \mathrm{~kW}$ a $45^{\circ} \mathrm{C}$, obteniéndose un COP de 1,48.

\section{REFERENCIAS}

[1] J. Ahn, H. Kang, H. Lee, H. Jung, Ch. Baek and Y. Kim. "Heating performance characteristics of a dual source heat pump using air and waste heat in electric vehicles". Applied Energy. Vol. 119, pp. 1-9. 2014.

[2] A. Yokoyama, T. Osaka, Y. Imanishi and S. Sekiya. "Thermal Management System for Electric Vehicles". SAE International Journal of Materials and Manufacturing. Vol. $4 \mathrm{~N}^{\circ} 1$, pp. 1277-1285. 2011.

[3] V. Pommé. "Reversible heat pump system for an electrical vehicle". SAE Technical Paper 971772. 1997. DOI: 10.4271/971772.

[4] T. Suzuki and K. Ishii. "Air Conditioning System for Electric Vehicle". SAE Technical Paper 960688. 1996.

[5] C. Cuevas, E. Winandy and J. Lebrun. "Testing and modeling of an automotive wobble plate compressor". International Journal of Refrigeration. Vol. 31, pp. 423-431. 2008.

[6] S.A. Klein. "Engineering Equation Solver". Fchart software, 2015.

[7] C. Cuevas, J. Lebrun, V. Lemort and $\mathrm{Ph}$. Ngendakumana. "Development and validation of a condenser three zones model". Applied Thermal Engineering. Vol. 29, pp. 3542-3551. 2009. 